\title{
An algorithm to construct nondominated k-coteries
}

\author{
Rikip Ginanjar, Nur Hadisukmana \\ Faculty of Computing, President University, Indonesia
}

\begin{tabular}{l}
\hline Article Info \\
\hline Article history: \\
Received Sep 1, 2019 \\
Revised Oct 23, 2019 \\
Accepted Nov 6, 2019 \\
\hline
\end{tabular}

\section{Keywords:}

Coterie

Mutual exclusion

Nondominated

Quorum

\begin{abstract}
One of the solution in solving $\mathrm{k}$ mutual exclusion problem is the concept of $\mathrm{k}$ coterie. A k-coterie under a set $\mathrm{S}$ is a set of subsets of $\mathrm{S}$ or quorums such that any $\mathrm{k}+1$ quorums, there are at least two quorums intersect each other. The $\mathrm{k}$ mutual exclusion problern is the problem of managing processes in such a way that at most $\mathrm{k}$ processes can enter their critical sections simultaneously. Nondominated k-coteries are more resilient to network and site failures than doninated k-coteries; that is the availability and reliability of a distributed system is better if nondominated k-coteries are used. Algorithms to construct $\mathrm{k}$-coteries have been proposed, unfortunately they have some restrictions, especially in constructing nondominated $\mathrm{k}$-coteries. The restrictions are due to the combination of $\mathrm{N}$, the number of nodes in a distributed system, and $\mathrm{k}$, the number of processes allowed to enter their critical sections simultaneously. To solve this problem, this paper proposes an algorithm to construct nondominated $\mathrm{k}$-coteries for all combination of $\mathrm{N}$ and $\mathrm{k}$.
\end{abstract}

Copyright (C) 2019 Institute of Advanced Engineering and Science. All rights reserved.

\section{Corresponding Author:}

Rikip Ginanjar,

Faculty of Computing,

President University, Indonesia.

Email: rikipginanjar@president.ac.id

\section{INTRODUCTION}

A distributed system may have shared resources which must be accessed in a mutually exclusive way. If a set of $\mathrm{k}$ identical resources may be simultaneously accessed by processes, it is said that multiple entries to critical sections are allowed. The concept of a coterie introduced by Garcia-Molina and Barbara [1] can be extended to be used in the distributed multiple mutual exclusion problems [2-7]. If a shared resource allows up to $\mathrm{k}$ processes to enter critical sections, it is called the $\mathrm{k}$-mutual exclusion problem. The distributed k-mutual exclusion problem is the problem of managing processes in a distributed system in such a way that at most $\mathrm{k}$ processes can enter their critical sections simultaneously. Several distributed k-mutual exclusion algorithms have been proposed [2, 8-23].

For example, suppose that there are $\mathrm{k}$ servers that contain identical license resources that are shared by nodes in a distributed system. Each license resource may only be accessed by one node at a time, and each node may access at most one license resource at a time [2, 24-25]. In this situation, a mutual exclusion algorithm can be used to control access to the servers.

Huang, Jiang, and Kuo [2] proposed the concept of a k-coterie, which is the extension of the concept of coterie introduced by Garcia-Molina and Barbara. The definition of a k-coterie proposed by Huang, Jiang, and Kuo satisfies two properties: intersection property and minimality property. Given a set of processes [nodes] $\mathrm{S}$ in the system, a k-coterie under $\mathrm{S}$ is a collection of subsets of $\mathrm{S}$ in which any $\mathrm{k}+1$ subsets have a nonempty intersection. This property is called the intersection property. The intersection property guarantees that at most $\mathrm{k}$ processes can enter their critical sections. The other property, minimality property, says that any two distinct quorums are not a subset of each other.

Independently, Kakugawa, Fujita,Yamashita, and Ac [3] proposed the same concept of a k-coterie. The definition for a k-coterie given by Kakugawa et. al. is more restrictive. Three properties must be satisfied: 
intersection property, minimality property, and non-intersection property. The non-intersection property assures that up to $\mathrm{k}$ processes can enter their critical sections. A subset of a k-coterie is called a quorum.

Garcia-Molina and Barbara [1] clasifled coteries into two categories: Dominated and Nondominated. Nondominated coteries are the most resilient coteries [24, 25]. Since the nondominated coteries are the most resilient, it is beneficial to find as general as possible an algorithm to constructing k-coteries. Some researchers have observed and analyzed the advantages of using nondominated coteries, such as [1, 16, 17, 20, 21].

\section{RESEARCH METHOD}

A k-coterie $\mathrm{C}$ is a set of subsets (also called quorums) of an underlying set of nodes, such that in any collection of $\mathrm{k}+1$ pairwise quorums there exists at least two quorums that intersect each other. This concept, introduced in two papers, in [2] and [3], independently, is an extension of the concept of a coterie. Huang, Jiang, and Kuo [2] defined a set of $\mathrm{C}$ to be a k-coterie if it satisfies two properties: intersection and minimality properties. Intersection property assures that at most $\mathrm{k}$ processes can enter their critical sections. Minimlaity property says that there is no quorum in $\mathrm{C}$ which is a subset of the others. The second paper was written by Kakugawa, Fujita, Yamashita, and Ae [3]. In the second paper, the definition of a k-coterie is more restrictive. A set $\mathrm{C}$ is said to be a k-coterie if it satisfies three properties: minimality, intersection, and non-intersection properties. First, consider the definition of a k-coteries proposed in [2].

\section{Definition 1. $k$-coterie}

Let $\mathrm{S}$ be a set of $\mathrm{N}$ nodes in the system and let $\mathrm{k}$ be a positive natural number $(\mathrm{k} \leq \mathrm{N})$, respectively. Then a set of subsets $\mathrm{C}$ which satisfies the following two conditions is called a k-coterie under S:

\section{Intersection Property.}

For any $\mathrm{k}+1$ set $\{\mathrm{Q} 1, \mathrm{Q} 2, \ldots, \mathrm{Qk}+1\} \subseteq \mathrm{C}$, there exist two elements $\mathrm{Qi}$ and $\mathrm{Qj}$ in $\mathrm{C}$ such that $\mathrm{Qi} \cap \mathrm{Qj}$ $\neq \varnothing$.

\section{Minimality Property.}

For any two distinct elements $\mathrm{Qi}$ and $\mathrm{Qj}$ in $\mathrm{C}, \mathrm{Qi} \not \subset \mathrm{Qj}$. The element $\mathrm{Q}$ of $\mathrm{C}$ is called a quorum.

In this part, a new class of $\mathrm{k}$-coteries is introduced: proper $\mathrm{k}$-coterie. A set $\mathrm{C}$ is called a proper $\mathrm{k}$ coterie when it satisfies three properties as proposed in [2] and [3]. The first two properties are exactly the same as in Definition 1. The third property is the non-intersection property.

\section{Definition 2. Proper k-coterie.}

A set $\mathrm{C}$ of subsets ( quorums ) is said a proper k-coterie if it is a k-coterie and satisfies the nonintersection property.

\section{Non-intersection Property.}

For any integer $h<k$, if an h-set $\{\mathrm{Q} 1, \mathrm{Q} 2, \ldots, \mathrm{Qh}\} \subseteq \mathrm{C}$ satisfies $\mathrm{Qi} \cap \mathrm{Qj} \neq \varnothing$, for all $i \neq j, 1 \leq i, j \leq h$, then there exists an element $Q \in C$, such that $Q \cap Q i=\varnothing$ for all $1 \leq i, j \leq h$.

The non-intersection property guarantees that even if $\mathrm{h}(<\mathrm{k})$ processes are in their critical section, another process can still enter its critical section.

\subsection{Proposed Algorithm \\ 2.1.1 Motivation}

In this paper, an algorithm to construct nondominated k-coteries for any combination of $\mathrm{N}$ (number of nodes in the system) and $\mathrm{k}$ (number of processes allowed to enter critical sections simultaneously) is proposed. Since the nondomnated coteries are the most resilient to network and node failures, it is beneficial to consider the construction algorithm producing nondominated k-coterie. The algorithm proposed works for every combination of $\mathrm{N}$ and $\mathrm{k}$. By constructing nondominated $\mathrm{k}$-coteries for any combination of $\mathrm{N}$ and $\mathrm{k}$ by this algorithm, the problems in the construction of coteries by the Cube, the Hypercube, and the Majk algorithms are solved for any value of $\mathrm{N}$ and $\mathrm{k}$. However, the algorithm may not construct so-called symmetric coteries.

The idea of this algorithm is to avoid some cases for which the Majk method cannot provide exactly a k-coterie. Moreover, this algorithm provides nondominated k-coteries for any combination of $\mathrm{N}$ and $\mathrm{k}$. The important thing of this algorithm is in reducing the number of votes for some nodes. 


\subsubsection{Algorithm}

Let $\mathrm{S}=\{\mathrm{a} 1, \mathrm{a} 2, \ldots, \mathrm{aN}\}$ be a set of $\mathrm{N}$ nodes in a distributed system where $\mathrm{N}$ is a non-negative integer. Let $\mathrm{k}$ be an integer, where $1<k<N$, representing the number of processes that can enter to their critical sections simultaneously

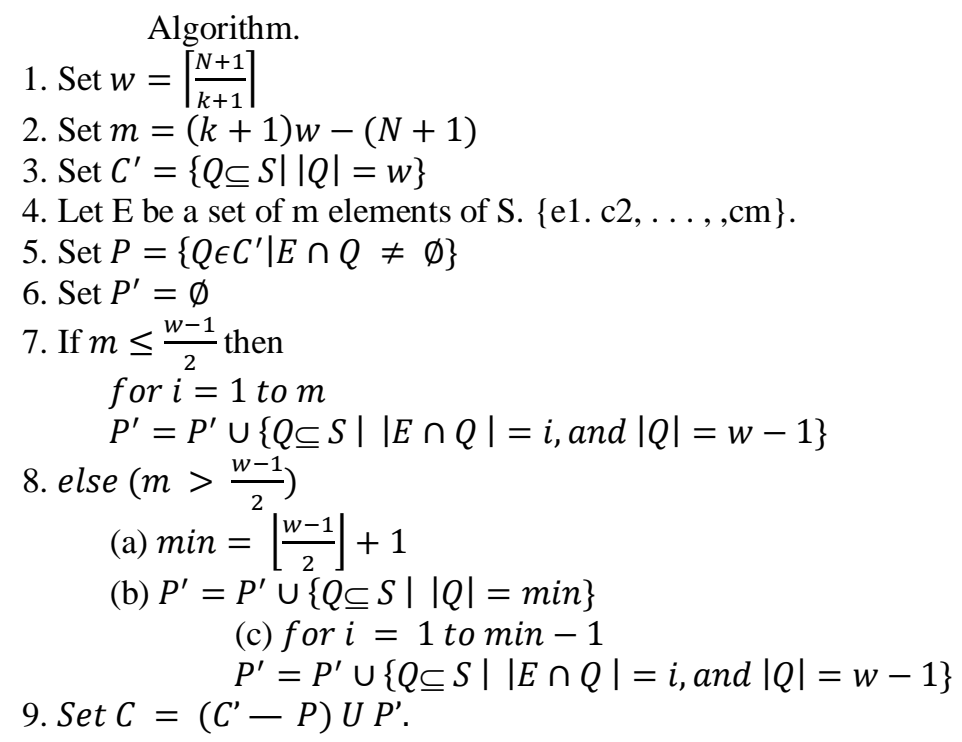

From the algorithm, quorums that contain exactly one element of a set $E=\left\{e_{1}, e_{2}, \ldots, e_{m}\right\}$ constructed by the algorithm have the size $(w-1)$. The quorums that contain two elements of $E$ have the size $(w-2)$. Generally speaking, the quorums that contain $\mathrm{h}$ elements of $\mathrm{E}$ have the size $(w-h)$, where $1<$ $h<\min$. There is an exception when $\mathrm{w}$ is odd, Since $\min =\left\lfloor\frac{\mathrm{w}-1}{2}\right\rfloor+1$, then $\min =\frac{\mathrm{w}+1}{2}$. We can see that $\mathrm{w}-\min <\min$. In this case, where $\mathrm{Q} \subseteq \mathrm{E}$, the quorums do not follow the above rule saying that quorums containing h elements of $\mathrm{E}$ have the size $(w-h)$.

Since $m=(k+1) w-(N+1)$, it is easy to see that $\mathrm{m} \leq \mathrm{k}$. It is also easy to see that any collection of $\mathrm{k}$ pairwise disjoint ciuorums contains at least $(N-m+1)$ nodes. Any $\mathrm{k}$ pairwise disjoint quorums contain exactly $N-w+1$ elements of $\mathrm{S}$ (nodes) if all quorums forming it have size ( $w-$ 1) $\operatorname{or}(N+1)-(k+1) n$, where $\mathrm{n}$ is an integer.

To show how the algorithm works, take a look at an example for $\mathrm{N}=6$, and $\mathrm{k}=2$. After step 3 , we get $\mathrm{w}=3, \mathrm{~m}=2$, and $\mathrm{C}^{\prime}=\{\{1,2,: 3\},\{1,2,4\},\{1,2.5\},\{1,2,6\},\{1,3,4\},\{1,3,5\},\{1,3,6\},\{1,4,5\},\{1,4$, $6\},\{1,5,6),\{2,3.4\},\{2,3,5\},\{2,3,6\},\{2,4,5\},\{2,4,6\},\{2,5,6\},\{3,4,5\},\{3,4,6\},\{3,5,6\} .\{4,5$, $6\}\}$.

Assume $E=\{1,2\}$, then after step 8, we have $P=\{\{1,2,3\},\{1,2,4\},\{1,2,5\},\{1,2,6\},\{1,3,4\},\{1$, $3,5\},\{1,3,6),\{1,4,5\},\{1,4,6\},\{1,5,6\},\{2,3,4\},\{2,3,5\},\{2,3,6\},\{2,4,5\},\{2,4,6\},\{2,5,6\}\}$ and $\mathrm{P}^{\prime}=\{\{1,2\},\{1,3\},\{1,4\},\{1,5\},\{1.6\},\{2,3\},\{2,4\},\{2,5\},\{2,6\}\}$.

Finally, we have a 2-coterie $C, C=\{\{1,2\},\{1,3\},\{1,4\},\{1,5\},\{1,6\},\{2,3\},\{2,4\},\{2,5\},\{2$, $6\},\{3,4,5\},\{3,4,6\},\{3,5,6\},\{4,5,6\}\}$.

It is easy to see that $\mathrm{C}$ is a 2-coterie and nondominated. It can also be seen that for any collection of 2 pairwise disjoint quorums $\{\mathrm{Q} 1, \mathrm{Q} 2\}$, the number of elements (nodes) involved is between $4(>N-w+1)$ and $6(<N)$. For $N=5$ and $k=3$, the algorithm produces

$\mathrm{C}=\{\{1\},\{2\},\{3,4\},\{3,5\},\{4,5\}\}$.

This set $\mathrm{C}$ is a 3 -coterie and even nondominated.

\section{RESULTS AND ANALYSIS}

The objective of this analysis process is to prove that the proposed algorithm is correct and produces nondominated k-coteires. But before proffing the correctness of the algorithm, some properties should be introduced to lead to the conclusion that $\mathrm{C}$ is a k-coterie. After the correctness of the algorithm is proven, the algorithm has to be analysed to demostrate that this algorithm also produces nondominated coteries $\mathrm{C}$, for any combination number of nodes and processes.

Before we get to the conclusion that the proposed algorithm is correct and produces nondominated algorithm, there are some properties (Lemma) that are obtained from the algorithm. Since the idea of the 
algorithm is to avoid most cases in which the Majk method produces dominated k-coterie and not-exactly kcoterie by selecting in special elements, we have interesting properties of $\mathrm{m}$.

Lemma 1. $m<k$.

Proof:

Assume $m>k$. Since $m$ and $k$ are integers, let $\mathrm{m}=\mathrm{k}+\mathrm{I}+\varepsilon$, for $\varepsilon>0$. From algorithm in step $2, \mathrm{~m}=$ $(\mathrm{k}+1) \mathrm{w}-(\mathrm{N}+1)$, so we have

This contradicts to $\left\lceil\frac{N+1}{k+1}\right\rceil=w$

$$
\begin{gathered}
N+1=(k+1) w-m \\
N+1=(k+1) w-(k+1+\varepsilon) \\
N+1=(k+1)(w-1)-\varepsilon \\
N+1 \leq(k+1)(w-1) \\
\lceil(N+1) /(k+1)\rceil \leq w-1
\end{gathered}
$$

Lemma 2. $m(w-1) \leq N$

Proof:

$$
\begin{aligned}
& \text { Since }\left\lceil\frac{N+1}{k+1}\right\rceil=w \text {, then } m(w-1)<\left\lceil\frac{N+1}{k+1}\right\rceil \text { or } \\
& (k+1)(w-1)<N+1 \\
& (k+1)(w-1) \leq N \\
& k(w-1) \leq N
\end{aligned}
$$

By Lemma $1, m(w-1) \leq N$. This completes the proof.

\section{Lemma 3.}

Proof:

Any collection of k pairwise disjoint quorums contains at least $N-w+1$ elements. consider.

Let $\mathrm{R}=\left\{\mathrm{Q}_{1}, \mathrm{Q}_{2}, \ldots, \mathrm{Q}_{\mathrm{k}}\right.$, be a collection of $\mathrm{k}$ pairwise disjoint quorums. There are two cases to

1. if $\frac{N+1}{k+1}=w$, where $w$ is an integer. The number of elements is $k w=N-w+1$.

2. if $\frac{N+1}{k+1}=w$ is not an integer. There are two cases to consider.

(a) $\left|E \cap\left(\cup_{i=1}^{k} Q_{i}\right)\right|=m$. There are two possibilities.

- $\quad$ There are $m$ quorums in $R$ that have size $(w-1)$. In other words, there are $m$ quorums in $R$ that contain exactly one element of $E$. The number of elements in $R$ is

$$
\begin{gathered}
m(w-1)+(k-m) w=k w-m \\
=k w-(k+1) w+(N+1) \\
=N-w+1
\end{gathered}
$$

- $\quad$ There are some quorums in $R$ that contain more than one element of $\mathrm{E}$, or $\left|Q_{i} \cap E\right|>1$, where $Q_{i} \in R$ Rand for some $1 \leq i \leq k$. Without loss of generality, assume there exists a qnorum $Q_{i} \in R$ that $\left|Q_{i} \cap E\right|=j$, for $1 \leq i \leq k$, and $j \geq 2$. There are two cases:

i. $Q_{i} \not \subset E$. By the algorithm, $\left|Q_{i}\right|=w-j$. Since there are $k$ pairwise disjoint quorums in $R$, then there exists $j-1$ quorums in $R$ that have size $w$. The number of elements included in the $j$ quorums equals to $w-j+(j-1) w=j(w-1)$. The average size of these quorums is $w-1$.

ii. $Q \subseteq E$. If $\min =w-j$ or if $w$ is even, then this case is the same as previous one. If $\min =w-$ $j+1$ or if $\mathrm{w}$ is odd, there exists $j$ quorums in $R$ that have size $w$. The number elements included in the $j+1$ quorums is $w-j+I+j(w)=w(j+1)-(j+1)+2$ and the average size of the $j+1$ quorulns is larger than $w-1$. 
Since any quorum $Q \in R$ such that $|Q \cup E|=j$, or $j-1$ or $\mathrm{j}$ quorums that have size $w$. then the average size of $m$ qurums in $R$ containing $m$ elements of $E$ at least $w-1$. Therefore, by previous proof, we have $\left|\cup_{i=1}^{k} Q_{i}\right|>N-w+1$.

(b) $\left|E \cap\left(U_{i=1}^{k} Q_{i}\right)\right|=m-j$. There are two possibilities.

- $Q_{i} \not \subset E$, for all $1<i<k$. By previous proof, the average size of the $m-j$ quorums is $w-1$. Consequently, the number of elements is $(m-j)(w-1)+(k-m+j) w=k w-m+j=N-$ $w+j+1$ •

- $Q \subseteq E$, for some $1<i<k$. By previous proof, the average size of the $m-j$ quorums is equal to or greater than $w-1$. Therefore, the number of elements in $R$ is equal to or greater than $N-w+$ $j+1$.

From the Lemma 2 and Lemma 3, there is always a collection of k disjoint quorums which contains $m$ disjoint quorumns having the sizes of $(m-1)$. Consequently, there is a collection of $\mathrm{k}$ pairwise disjoint quorums with $m$ disjoint quorums having the sizes of $(m-1)$ and $(k-m)$ disjoint quorums having the sizes of $w$.

Lemma 4. elements.

There exists a collection of $\mathrm{k}$ pairwise disjoint quorums in $\mathrm{C}$ that consists exactly of $N-w+1$

Proof:

From Lemma 2, we get $m(w-1) \leq N$, or $m(w-2) \leq N-m$. This means that there are $(N-$ $m$ ) elements winch are sufficient enough to form a collection of $m$

pairwise disjoint quorums that each quorum has the size of $(w-1)$. Then by Lemma $3, m(w-1)+$ $(k-m) w=N-w+1$.

From Lemma 1, Lemma 2, and Lemma 3, we obtain the following Theorem 2.

Theorem 2. $C$ is a k-coterie under $S$.

Proof:

To prove the Theorem, we have to show that $\mathrm{C}$ has two properties: intersection and minimality properties.

1. Minimality Property. It is obvious, from algorithm that every quorum produced complies the minimality property, since for two distinct quorums and $Q_{i}, Q_{j}, Q_{i} \not \subset Q_{j}$.

2. Intersection Property. Let $R=\left\{Q_{1}, Q_{2}, \ldots, Q_{k}\right\}$ be a collection of $k$ pairwise disjoint quorums and let $Q \in C$ be another distinct quorum. There are two cases to consider:

- $\left|Q_{i}\right|=w$, for $1<i<k$. This means that $k w<N-m$. Since $w=(k+1) w-(N+1)$, it implies that $k w=m+N+1-w \leq N-m$ or $m \leq \frac{w-1}{2}$. By the algorithm, the smallest quorums produced have size $w-m$. Then, the number of elements of $k+1$ pairwise quorums is $k w+w-$ $m=N+1>N$.

- $\left|Q_{i}\right|<w$ for some $1<i<k$. This means that some elements of $E$ are included in $R$. Assume in $m-j$ elements of $E$ are included in $R$, where $0 \leq j \leq m$, then the number of elements is, by Lemma 3, at least $N-w+j+1$. In this case, there are two possibilities:

1) $j \geq \min$. Then, the smallest size of $\mathrm{Q}$ or $|Q|=\min$ and $w-j \leq \min$. Therefore, the number of elements in $\mathrm{R}$ is $N-w+j+1+\min =N-(w-j)+\min +1 \geq N+1>N$.

2) $j<\min$. Then the smallest $|Q|=w-j(>\min )$. The number of elements is $N-w+j+I+$ $w-j=N+1>N$. each other.

Consequently. in any $k+1$ pairwise quorums, there exists at least two quorums that intersect

Theorem 3. $C$ is a proper k-coterie under $S$ if one of the following properties is satisfied:

1. $w$ is even.

2. $w$ is odd and $m<2 w$.

Proof:

1. First, we will prove that if $w$ is even, $\mathrm{C}$ satisfies the non-intersection property. From Lemma 3 , if $\left|E \cap\left(\cup_{i=1}^{k-1} Q_{i}\right)\right|=m-j$, the average size of the $m-j$ quorums is $w-1$. The number of elements in a collection of $k-1$ pairwise disjoint quorums is $(k-1) w-m+j$. Let $f(j)=(k-1) w-m+j$. We can easily see that $f(j)$ is a monotone increasing function.

- If $j=m$, then $f(j)=(k-1) w$ and $f(j) \leq N-m$. We can form another quorum $Q \subseteq C$ and $|Q|=\min$. 
- If $j=0$, then $f(j)=(k-1) w-m$. By Lemma 3, we can find another quorum so that the number of the collection of $k$ pairwise disjoint quorums is $N-w+1$.

This completes the proof for number 1 .

2. Second, if $\mathrm{w}$ is odd, and $\mathrm{m}<2 \mathrm{w}, \mathrm{C}$ satisfies the non-intersection property. The only difference from the previous one is when the collection of $\mathrm{k}-1$ disjoint quorums contains all possible quorums $\mathrm{Q}$, where $\subseteq \mathrm{E}$ . Since $\mathrm{w}$ is odd, then $\min =\frac{\mathrm{w}+1}{2}$ The number of quorums $\mathrm{Q} \subseteq \mathrm{E}$ is $\left\lfloor\frac{2 m}{w+1}\right\rfloor$ Since $\mathrm{m} \leq 2 \mathrm{w}-1$ then we have

$$
\begin{gathered}
\frac{2 m}{w+1} \leq \frac{2(2 w-1)}{w+1} \\
\left.\frac{2 m}{w+1} \leq \frac{2(w+1)}{w+1}+\frac{2(w-2}{w+1}\right) \\
\left\lfloor\frac{2 m}{w+1}\right\rfloor \leq 3
\end{gathered}
$$

The number of other elements (nodes) is $N-m$. These elements can form other quorums. The number of quorums that can be formed from these elements is $\left\lfloor\frac{N-m}{w}\right\rfloor$. Since $N=(k+1) w-(m+1)$ and $m<$ $2 w-1$, then we have

$$
\begin{gathered}
\frac{N-m}{w}=\frac{(k+1) w-(m+1)-m}{w} \\
\frac{N-m}{w}=\frac{(k+1) w-(2 m+1)}{w} \\
\frac{N-m}{w} \geq(k+1)-\frac{2(2 w-1)+1}{w} \\
\frac{N-m}{w} \geq(k+1)-\frac{4 w-1}{w} \\
\lfloor(N-m) / w\rfloor \geq k-3
\end{gathered}
$$

This implies that the non-intersection property is satisfied.

By Theorem 2, $C$ is $k$-coterie under $S$. Because $C$ also satisfies the non-intersection property, it can be concluded that $C$, with above two conditions, is a pi'oper $k$-coterie under $S$.

Theorem 4. $C$ is a nondominated $k$-coterie under $S$.

Proof:

Since any collection of $\mathrm{k}$ pairwise disjoint quorums $\left\{Q_{1} Q_{2}, \ldots, Q_{k}\right\}$ contains $x$ elements, where $x \geq$ $N-w+1$, there is no subset $H$ of $S$ satisfying Theorem 1 . Assume that $C$ is a dominated coterie. By Theorem 1, there exists a subset $H \subseteq S$ satisfying two conditions:

1. for every $Q \in C, Q \not \subset H$, and

2. for ally collection of k pairwise disjoint quorums $\left\{Q_{1}, Q_{2}, \ldots, Q_{k}\right\} \subseteq C, H \cap Q_{i} \neq \emptyset$, for some $i, 1<i<$ $k$. The size of $H$ must be less than or equal to $(w-1)$. There are two possibilities of the size of $H$ or $|H|$

a. If $\min \leq|H| \leq w-1$, then $H$ must be a subset of some quorums which do not contain any element of a set $E=\left\{e_{1}, e_{2}, \ldots, e_{k}\right\}$. But then, this $H$ does not satisfy the second condition of Theorem 1 , since by Lemma 3 and Lemma 4, we can always select a collection of $k$ pairwise quorums that contains exactly $N-w+1$ elements that do not include any element of the subset. $H \subseteq S$.

b. If $|H|<\min$, then, again, this $H$ cannot satisfy the secoiid condition of the Theorem 1 . There are two cases 
1) If $H \cap E=\emptyset$. This means that $H$ is a subset of a collection of quorums that do not consist of any element of set $E$. By the previous proof, $H$ does not satisfiy the second condition of Theorem 1 .

2) (h) If $H \cap E \neq \varnothing$. Form a set of $k$ pairwise disjoint quorums which consists of $\left(m-\left\lfloor\frac{w-1}{2}\right\rfloor\right)$ quorums that have sizes of $(w-1)$ and $\left(k-\left(m-\left\lfloor\frac{w-1}{2}\right\rfloor\right)\right.$ quorums that have sizes of $w$. Let

then we have

$$
\left.f\left(m-\left\lfloor\frac{w-1}{2}\right\rfloor\right)\right)=\left(m-\left\lfloor\frac{w-1}{2}\right\rfloor\right)(w-I)+\left(k-\left(m-\left\lfloor\frac{w-1}{2}\right\rfloor\right) w .\right.
$$

$$
\begin{gathered}
f\left(m-\left\lfloor\frac{w-1}{2}\right\rfloor\right)=k w-\left(m-\left\lfloor\frac{w-1}{2}\right\rfloor\right) \\
f\left(m-\left\lfloor\frac{w-1}{2}\right\rfloor\right)=k w-\left((k+1) w-(N+1)-\left\lfloor\frac{w-1}{2}\right\rfloor\right) \\
\left.f\left(m-\left\lfloor\frac{w-1}{2}\right\rfloor\right)=N-\left\lfloor\frac{w-1}{2}\right\rfloor\right) \\
\left.f\left(m-\left\lfloor\frac{w-1}{2}\right\rfloor\right)=N-\min +1\right)
\end{gathered}
$$

Again, since we can form a collection of $\mathrm{k}$ pairwise disjoin quorums that contains at most $-\min +1, H$ does not satisfy the second condition of Theorem 1 .

It can be concluded there is no $H$ satisfying two conditions of Theorem 1 . So $C$ is nondominated $k$-coterie.

\section{CONCLUSION}

The algorithm to construct nondominated k-coteries is presented. This algorithm works for any combination of $\mathrm{N}$, the number of nodes in a distributed system, and $\mathrm{k}$, the number of processes allowed to enter critical sections symultaneously. The algorithm is the extension of Majk method, which produces mainly dominated k-coteries. The proposed algorithm also produces nearly symmetric k-coteries. Although the algorithm to construct nondominated k-coteries for any value of $\mathrm{N}$ and $\mathrm{k}$, has been proposed, there is still an open challenging problem. The problem found here is how to find an algortihm that can construct symmetric and proper nondominated k-coteries. What we have here is that still our algorithm may not construct symmetri $\mathrm{k}$-coteries especially when $(\mathrm{N}+1) /(\mathrm{k}+1) \neq \mathrm{w}$. But, the algorithm may not produce proper coteries when $\mathrm{w}$ is odd and $\mathrm{m}>2 \mathrm{w}$.

\section{REFERENCES}

[1] H. Garcia-Monila and D. Barbara. How to assign votes in a distributed system. Journal of A CM, 32(4):841-860, 1985

[2] S.T. Huang, .J.R. Jiang, and Y.C. Kuo. K-coteries for fault $k$ entries to a critical section. IEEE 13th International Conference on Disiributed Computing Systems, pages 74-81, 1993.

[3] H. Kokugawa, S. Fujita, M. Yamashita, and T. Ae. A distributed k-mutual exclusion algorithm using k-coterie. 2nd International Symposium on Algorithms (LNCS 557), pages 22-:31, 1991.

[4] H. Kokugawa, S. Fujita, M. Yamasluta, and T. Ac. Availability of kcoterie. IEEE Transactions on Computers, 42(5):553-55S, 1993.

[5] D. Agrawal and A. El Abbadi, a An Efficient and Fault-Tolerant Solution for Distributed Mutual Exclusion, ACM Trans. Computer Systems, vol. 9, no. 1, pp. 1-20, Feb. 1991.

[6] S. Fujita and M. Yamashita. Constructing asymptotically optimal k-coterie. In The Proceeding of Th 2nd International Syniposium on Algorithms, 1991.

[7] D. Barbara and H. Garcia-Molina. The reliability of voting mechanisms. IEEE Trans actions on Computers, C36(1O):1 197-1208, 1987.

[8] M.L. Neilsen and M. Mizuno. Coterie join algorithm. IEEE Transactions on Parallel and Distributed Systems, 3(5):582-590, 1992.

[9] T. Ibaraki and T. Kaineda. A theory of coteries: Mutual exclusion in distributed systems. IEEE Transactions on Parallel and Distributed Systems. 4(7):779-793, 1993.

[10] Yu-Chen Kuo and Po-Yao Wu. The Availability of Complemental k-Coteries. The Computer Journal, Vol. 51 No. 6, 2008.

[11] S. T. Huang, J.R. Jiang and Y.C Kuo. IEEE Transaction on Computers. Vol 46 issue 2. Pages 75-81. February 1997 
[12] Rodrigues, Luiz, Duarte Jr., Elias \& Arantes, Luciana: A Distributed k-mutual exclusion algorithm based on autonomic spanning trees. Journal of Parallel and Distributed Computing.115. 2018

[13] T. Harada and M. Yamashita. K-Coteries for Tolerating Network 2-Partition. IEEE Transaction on Parallel and Distributed Systems. Vol 15 No. 7, pages 666-672. July 2004

[14] H.K. Chang, S.M. Yuan, Performance Characterization of the Tree Quorum Algorithm, IEEE Trans. Parallel and Distributed Systems, vol. 6, no. 6, pp. 658-662, June 1995.

[15] E.H. Chot, T. Tsuchiya and T.Kikuno. New Constructions for Nondominated k-Coteries. IEICE Trans. Inf \& Syst. Vol E83-D. No. 7. Pages 1526-1532. July 2000

[16] Y.-C. Kuo and S.-T. Huang, A Geometric Approach for Constructing Coteries and k-Coteries, IEEE Trans. Parallel and Distributed Systems, vol. 8, no. 4, pp. 402-411, Apr. 1997.

[17] La Ode Muhlis, Armin Lawi, Amir Kamal Amir. Operasi Join Koteri-k Diperluas. Jurnal Matematik. Statistik dan Komputasi. Vol 14 No.2, pages 106-113. 2018

[18] J.-R. Jiang, Distributed h-out of-k mutual exclusion using k-coteries, in Proc. of the 3rd International Conference on Parallel and Distributed Computing, Application and Technologies (PDCAT’02), pp. 218-226, 2002.

[19] H. Kakugawa and M. Yamashita, Local coteries and a distributed resource allocation algorithm, Transactions of Information Processing Society of Japan, 37(8):1487-1496, 1996

[20] Yuh-Jzer Joung. On Quorum Systems for Group Resources with Bounded Capacity. Proceedings Distributed Computing, 18th International Conference, DISC 2004, Amsterdam, The Netherlands, October 4-7, 2004

[21] Jehn-Ruey Jiang, Cheng-Sheng Cho, Shing-Tsaan Huang. Constructing nondominated local coteries for distributed resource allocation. International Conference on Parallel Processing Workshops, 6-9 Oct. 2003.

[22] A. Lawi, K. Oda, T. Yoshida. A Quorum Based Group k-Mutual Exclusion Algorithm for Open Distributed Environments. International Symposium on Parallel and Distributed Processing and Applications. Nanjing, China, November 2-5, 2005. pp 119-125

[23] Jehn-Ruey Jiang, Shing-Tsaan Huang. Obtaining nondominated k-coteries for fault-tolerant distributed k-mutual exclusion. Proceedings of 1994 International Conference on Parallel and Distributed Systems. Hsinchu, Taiwan, Taiwan .19-21 Dec. 1994

[24] M.L. Neilsen and M. Mizuno. Nondominated k-coterie for multiple exclusion. Information Processing Letters, 50(5):247-252, 1994

[25] Mitchell L.Neilsen. Properties of nondominated k-coteries. Journal of Systems and Software. Volume 37, Issue 1, April 1997, Pages 91-96 